\title{
Integrative cancer pharmacogenomics to establish drug mechanism of action: drug repurposing
}

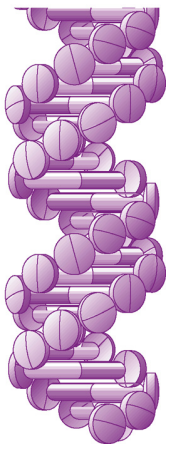

\author{
Nehme El-Hachem ${ }^{1}$, Wail Ba-alawi ${ }^{1,2}$, Ian Smith ${ }^{1,2}$, Arvind Singh Mer ${ }^{1,2}$ \& Benjamin \\ Haibe-Kains*, 1,2,3,4 \\ 1 Princess Margaret Cancer Centre, University Health Network, Toronto, Ontario, Canada \\ ${ }^{2}$ Department of Medical Biophysics, University of Toronto, Toronto, Ontario, Canada \\ ${ }^{3}$ Department of Computer Science, University of Toronto, Toronto, Ontario, Canada \\ ${ }^{4}$ Ontario Institute of Cancer Research, Toronto, Ontario, Canada \\ * Author for correspondence: bhaibeka@uhnresearch.ca
}

"With the availability of large-scale pharmacogenomic data and new computational methods to leverage these complex data, a new era of innovations in drug repurposing lies ahead of us."

First draft submitted: 24 July 2017; Accepted for publication: 24 July 2017; Published online: 23 October 2017

Keywords: cancer $\bullet$ drug repurposing $\bullet$ integrative analysis • machine learning • pharmacogenomics

A total of 100 years ago, Paul Ehrlich, a Nobel Laureate in physiology and medicine, proposed the concept of 'magic bullets' - that is, chemical compounds precisely interacting with a specific receptor. For decades, the 'one drug one target - one disease' paradigm dictated much of the drug development process. However, in the past 10 years, tremendous advances in transcriptomics and genomics research have shifted this simplistic view of mechanism of action $(\mathrm{MoA})$ to a more complex systems pharmacology paradigm where a compound can bind to several targets.

Experimental elucidation of drug MoA constitutes a critical element in the early preclinical drug development process. This step is labor intensive, costly and very complex since many drug-target interactions (DTI) remain elusive $[1,2]$. Biochemical assays and genetic interaction approaches were traditionally used to understand MoA of drug candidates [3]. However, these experimental approaches are time consuming. The increasing availability of computational resources and public biochemical assay and pharmacogenomic data has enabled the development of computational drug-target identification strategies over their more traditional experimental counterparts. Computational approaches are categorized into two main categories: chemoinformatics, which includes structure-based virtual screening or molecular docking and ligand-based structural similarity approach; and systems biology, which focuses more on biological and chemogenomic data [4].

Chemoinformatics mainly relies on structure-based protein ligand docking which requires a three-dimensional structure of a protein and a library of small molecules and uses sophisticated computational tools in order to model the docking of a ligand into a protein binding pocket [5]. However, many protein structures are unavailable. Ligand-based similarity approaches assume that two similar protein sequences bind similar drugs, so for a given small drug with a known protein partner, a highly similar protein would be predicted as a putative target for the tested drug [6]. The limitation of this method is that it is only applicable to drugs with established binding information toward corresponding targets which is not the case for chemical compounds in early screening phases.

In contrast, systems-based approaches leverage a diverse set of high-dimensional pharmacogenomic data. Some of these methods [7] infer new DTI from comprehensive pharmacological and structural data regarding known DTI from online resources, such as DrugBank [8] and PubChem [9]. Cheng et al. developed a network-based inference method that predicts a new DTI using a two-step diffusion process on a bipartite graph of drugs and targets, and their known interactions as edges [10]. Other methods have extended this seminal work with sophisticated graph traversal methods to infer new DTI [11,12]. These methods have shown improved accuracy and provided better scalability than structure- and ligand-based approaches. However, they usually rely on high-level annotations of drugs and targets, such as 2D chemical structure and amino acid sequence, respectively. The establishment of the

Future $\mathrm{s}$ Medicine 
connectivity map concept [13] paved the way for pioneering strategies that have used drug-induced transcriptional perturbations to infer drug-drug similarity networks for drug repurposing [14]. To scale up the initial connectivity map dataset, the NIH Library of Integrated Network-Based Cellular Signatures (LINCS) consortium developed the low-cost L1000 transcriptional assay, enabling interrogation of 1000 gene expressions perturbed by thousands of compounds and genomic reagents across tens of cell lines [15]. The resulting set of perturbation signatures can be used to infer similarities among small molecules as edges in a network and the resulting adjacency matrices can be further mined with network algorithms to identify classes of drugs and compounds. The NIH LINCS drug repurposing hub is a library of approved drugs and compounds in stages of development that have been curated and extensively annotated [16]. Drug repurposing hub uses a systems biology approach based on structural similarities and phenotypic assays to characterize drug function. To further elucidate function, a high-throughput compound viability screen against barcoded panels of cell lines (PRISM), which when coupled with biomarker discovery methods from previous drug sensitivity panels can elucidate function [17].

Although these systems-based approaches represent substantial advances in the drug-target predictive field, the current drug taxonomies they proposed rely on information, difficult to gather for new compounds (e.g., DTI), which limits their utility for early drug development. Moreover, these approaches integrate a limited number of data layers, despite their high complementarity $[18,19]$. This motivated us to develop Drug Network Fusion (DNF), a computational method combining diverse types of drug-centered data to improve drug taxonomy for inference of drug target and MoA [20].

\section{DNF to pinpoint novel drug indications \& MoA in the drug development process}

DNF is the first tool to integrate pharmaco-chemo-genomics high-throughput data, including chemical structures, pharmacological growth inhibition profiles and post-treatment transcriptional perturbations, to develop comprehensive drug taxonomy [20]. DNF leverages PharmacoGx, our R/Bioconductor package including the largest pharmacogenomic datasets published to date [21]. The first data layer used in DNF is the structural information or the 'chemical space' available through PubChem, a database with more than 60 million chemical structures [22]. The SMILES string for each chemical provides sufficient information to assess structural similarity between two chemicals using a generic metric, the Tanimoto index [23]. For the second data layer, DNF capitalizes on the recent L1000 dataset composed of 1.8 M gene expression profiles pre- and post-treatment for over 20,000 chemical compounds [15]. These transcriptional perturbation data are used to identify genes that are significantly down and upregulated due to drug treatment, referred to as drug perturbation signatures [20,21]. These signatures can in turn be used to find compounds with similar effect on the cell's transcriptome. At last, DNF integrates growth inhibition measurements across cancer cell lines that are publicly available from NCI60 [24] and CTRPv2 [25].

Although each data layer can be used to generate a 'drug-drug' similarity matrix to predict which chemical compounds have similar targets or MoA, we sought to integrate them to further improve the accuracy of such drug taxonomy. We therefore, applied the recent similarity network fusion algorithm [26] to fuse the three drug-drug similarity matrices described previously. To validate the performance of DNF versus single layers, we tested the predictive value against gold standard benchmarks: DrugBank for drug-targets associations [27] and ChEMBL for anatomical therapeutic classification [28]. We found that DNF significantly outperformed all single layers and showed the highest accuracy in detecting drug pairs with the same MoA when using both drug-target and anatomical therapeutic classification benchmarks [20].

Since our integrative approach yielded an improved drug taxonomy, we assessed the biological relevance of DNF using a clustering technique known as affinity propagation clustering [29]. This clustering approach was tuned to generate nonredundant communities of drugs. DNF classified correctly the major classes of drugs, including: HDAC inhibitors, statins, major receptor and nonreceptor protein tyrosine kinases inhibitors, proteasome and PI3K/MTOR inhibitors. Furthermore, DNF results concurred with experimental findings for Bcl-2 inhibitors: ABT-737 and navitoclax that showed less off-target effect than obatoclax towards Bcl-2. Besides improving drug classification, a major application of DNF is drug repurposing. A relevant example is the clustering of ibrutinib, a Bruton tyrosine kinase inhibitor, with the EGFR inhibitor cluster set. Ibrutinib has showed activity against EGFR mutation [30]; thus, it might be useful against some cases of non-small-cell lung cancer resistant to EGFR inhibitors. Furthermore, DNF can enlighten about new potential targets. For example,we could recapitulate experimental findings showing that tivantinib, a MET kinase inhibitor, targets microtubule polymerization [31]. The main advantage of DNF compared with other drug repurposing strategies is that it relies solely on data that are relatively 
simple to generate experimentally, as we showed that transcriptional perturbation signatures and growth inhibition profiles for a few cell lines are enough to classify compounds according to their target and MoA.

With the availability of large-scale pharmacogenomic data and new computational methods to leverage these complex data, a new era of innovations in drug repurposing lies ahead of us. While most drug repurposing approaches rely on information difficult to gather, such as DTI, ligand-based similarities or side effects, new methods can be designed to classify experimental, poorly characterized compounds by integrating relatively basic experimental data. We have demonstrated the feasibility of integrating different drug-centered data for a better prediction of drug mode of action using our DNF algorithm. The expansion of large-scale pharmacogenomic datasets, makes our model attractive for further studies and validation. We are confident that our integrative approach constitutes a potential companion tool for medicinal chemists and drug scientists interested in drug MoA at early preclinical phases.

Financial \& competing interests disclosure

$\mathrm{N}$ El-Hachem was supported by the Ministry of Economic Development, Employment and Infrastructure and the Ministry of Innovation of the Government of Ontario. W Ba-alawi is supported by the Terry Fox Research Institute. Arvind Singh Mer is supported by the Canadian Institutes of Health Research and the Natural Sciences and Engineering Research Council of Canada. B Haibe-Kains was supported by the Gattuso-Slaight Personalized Cancer Medicine Fund at Princess Margaret Cancer Centre and the Canadian Institutes of Health Research. The authors have no other relevant affiliations or financial involvement with any organization or entity with a financial interest in or financial conflict with the subject matter or materials discussed in the manuscript apart from those disclosed.

No writing assistance was utilized in the production of this manuscript.

\section{References}

1 Chan JNY, Nislow C, Emili A. Recent advances and method development for drug target identification. Trends Pharmacol. Sci. 31(2), 82-88 (2010).

2 Gresham V, McLeod HL. Genomics: applications in mechanism elucidation. Adv. Drug Deliv. Rev. 61(5), 369-374 (2009).

3 Schenone M, Dančík V, Wagner BK, Clemons PA. Target identification and mechanism of action in chemical biology and drug discovery. Nat. Chem. Biol. 9(4), 232-240 (2013).

4 Dudley JT, Deshpande T, Butte AJ. Exploiting drug-disease relationships for computational drug repositioning. Brief. Bioinform. 12(4), 303-311 (2011).

5 Li H, Gao Z, Kang L et al. TarFisDock: a web server for identifying drug targets with docking approach. Nucleic Acids Res. 34(Web Server issue), W219-W224 (2006).

6 Keiser MJ, Setola V, Irwin JJ et al. Predicting new molecular targets for known drugs. Nature. 462(7270), 175-181 (2009).

7 Chen X, Yan CC, Zhang X et al. Drug-target interaction prediction: databases, web servers and computational models. Brief. Bioinform. 17(4), 696-712 (2016).

8 Law V, Knox C, Djoumbou Y et al. DrugBank 4.0: shedding new light on drug metabolism. Nucleic Acids Res. 42(Database issue), D1091-D1097 (2014).

9 Wang Y, Bryant SH, Cheng T et al. PubChem BioAssay: 2017 update. Nucleic Acids Res. 45(D1), D955-D963 (2017).

10 Cheng F, Liu C, Jiang J et al. Prediction of drug-target interactions and drug repositioning via network-based inference. PLoS Comput. Biol. 8(5), e1002503 (2012).

11 Chen X, Liu MX, Yan GY. Drug-target interaction prediction by random walk on the heterogeneous network. Mol. Biosyst. 8(7), 1970-1978 (2012).

12 Ba-Alawi W, Soufan O, Essack M, Kalnis P, Bajic VB. DASPfind: new efficient method to predict drug-target interactions. J. Cheminform. 8(1), 15 (2016).

13 Lamb J, Crawford ED, Peck D et al. The connectivity map: using gene-expression signatures to connect small molecules, genes, and disease. Science. 313(5795), 1929-1935 (2006).

14 Iorio F, Bosotti R, Scacheri E et al. Discovery of drug mode of action and drug repositioning from transcriptional responses. Proc. Natl. Acad. Sci. USA 107(33), 14621-14626 (2010).

15 Subramanian A, Narayan R, Corsello SM, Peck DD. A next generation connectivity map: L1000 platform and the first 1,000,000 profiles. bioRxiv. doi.org/10.1101/136168 (2017) (Epub ahead of print).

16 Corsello SM, Bittker JA, Liu $\mathrm{Z}$ et al. The drug repurposing hub: a next-generation drug library and information resource. Nat. Med. 23(4), 405-408 (2017). 
17 Yu C, Mannan AM, Yvone GM et al. High-throughput identification of genotype-specific cancer vulnerabilities in mixtures of barcoded tumor cell lines. Nat. Biotechnol. 34(4), 419-423 (2016).

18 Chen B, Greenside P, Paik H, Sirota M, Hadley D, Butte AJ. Relating chemical structure to cellular response: an integrative analysis of gene expression, bioactivity, and structural data across 11,000 compounds. CPT Pharmacometrics Syst. Pharmacol. 4(10), 576-584 (2015).

19 Ma’ayan A, Rouillard AD, Clark NR, Wang Z, Duan Q, Kou Y. Lean big data integration in systems biology and systems pharmacology. Trends Pharmacol. Sci. 35(9), 450-460 (2014).

20 El-Hachem N, Gendoo DMA, Ghoraie LS et al. Integrative cancer pharmacogenomics to infer large-scale drug taxonomy. Cancer Res. 77(11), 3057-3069 (2017).

21 Smirnov P, Safikhani Z, El-Hachem N et al. PharmacoGx: an R package for analysis of large pharmacogenomic datasets. Bioinformatics 32(8), 1244-1246 (2016).

22 Kim S, Thiessen PA, Bolton EE et al. PubChem substance and compound databases. Nucleic Acids Res. 44(D1), D1202-D1213 (2016).

23 Rogers DJ, Tanimoto TT. A computer program for classifying plants. Science. 132(3434), 1115-1118 (1960).

24 Shoemaker RH. The NCI60 human tumour cell line anticancer drug screen. Nat. Rev. Cancer. 6(10), 813-823 (2006).

25 Seashore-Ludlow B, Rees MG, Cheah JH et al. Harnessing connectivity in a large-scale small-molecule sensitivity dataset. Cancer Discov. 5(11), 1210-1223 (2015).

26 Wang B, Mezlini AM, Demir F et al. Similarity network fusion for aggregating data types on a genomic scale. Nat. Methods. 11(3), 333-337 (2014).

27 Wishart DS, Knox C, Guo AC et al. DrugBank: a knowledgebase for drugs, drug actions and drug targets. Nucleic Acids Res. 36(Database issue), D901-D906 (2008).

28 Gaulton A, Bellis LJ, Bento AP et al. ChEMBL: a large-scale bioactivity database for drug discovery. Nucleic Acids Res. 40(Database issue), D1100-D1107 (2012).

29 Frey BJ, Dueck D. Clustering by passing messages between data points. Science 315(5814), 972-976 (2007).

30 Gao W, Wang M, Wang L et al. Selective antitumor activity of ibrutinib in EGFR-mutant non-small cell lung cancer cells. J. Natl. Cancer Inst. 106(9), pii:dju204 (2014).

31 Katayama R, Aoyama A, Yamori T et al. Cytotoxic activity of tivantinib (ARQ 197) is not due solely to c-MET inhibition. Cancer Res. 73(10), 3087-3096 (2013). 